\title{
Optimal and isodual ternary cyclic codes of rate 1/2
}

\author{
Cherif Mihoubi • Patrick Solé
}

Received: 12 January 2012 / Revised: 9 May 2012 / Accepted: 4 July 2012 / Published online: 26 July 2012 C The Author(s) 2012. This article is published with open access at SpringerLink.com

\begin{abstract}
This work is twofold. First, the largest minimum distance of a ternary cyclic codes of parameters $\left[n, \frac{n}{2}\right]$, is determined for $n$ even, not a multiple of 3 , by using the Chen algorithm, for $n=26,34,38,46,50,58,62,68,70,74$. Next, seven new classes of isodual ternary cyclic codes are introduced for $n$ singly even, not a multiple of 3 .
\end{abstract}

Keywords Cyclic codes $\cdot$ Minimum distance $\cdot$ Isodual codes

Mathematics Subject Classification (2000) 94B15 94 B05 94 B065

\section{Introduction}

An important class of ternary codes of rate $1 / 2$ is that of self-dual codes [5], because of its connections with invariant theory, combinatorial designs, and modular forms.

Communicated by S.K. Jain.

C. Mihoubi $(\varangle)$

Département de Mathématiques, Université Hadj Lakhdar, 5000 Batna, Algeria

e-mail: cherif.mihoubi@yahoo.fr

C. Mihoubi

Université de M'sila, Bp 581, Hodna, 28000 M'sila, Algeria

P. Solé

Telecom ParisTech, 46 rue Barrault, 75634 Paris Cedex 13, France

P. Solé

Mathematics Department, King Abdulaziz University, Jeddah, Saudi Arabia

e-mail: sole@ telecom-paristech.fr 
This class is a subclass of formally self dual codes in [2]. In the present work, we consider cyclic ternary codes of rate $1 / 2$. An important subclass of these is that of cyclic isodual codes, i.e. cyclic codes equivalent to their duals. Isodual codes are in particular formally self dual.

Following [2] we consider the largest minimum distance $d_{F}(n)$ of a fsd ternary code of length $n$. We introduce $d_{I}(n)$ the largest minimum distance of an isodual code of length $n$. Clearly $d_{I}(n) \leq d_{F}(n)$. Another function of interest is the largest minimum distance $d_{C}(n)$ of a cyclic code of length $n$ and rate $1 / 2$.

Our contribution is twofold. First, we establish by electronic calculation, using an algorithm due to Chen, the value of $d_{C}(n)$ for $n$ even $\leq 74$. Second, inspired by this numerical data, we propose, in the case $n=2 m$ with $m$ odd, seven constructions of ternary isodual cyclic codes. They hold when $n$ is singly even, that is at lengths where self dual ternary codes do not exist. The characterization of the generating polynomial of an isodual cyclic code is left as a challenging open problem.

The material is organized as follows. The next section reviews the necessary notation and definitions. Section 3 derives the seven constructions of isodual cyclic codes and gives a table of values of $d_{I}(n)$ and $d_{C}(n)$ for small values of $n$. Section 4 contains the numerical data on cyclic codes of rate $1 / 2$ over $\mathbb{F}_{3}$, arranged in subsections by values of the length.

\section{Notation and definitions}

We assume that the reader has gained some familiarity with coding theory $[1,4]$. Let $\mathbb{F}_{3}=\{0,1,2\}$ denote the Galois field of three elements. Recall that the rate of a linear code of length $n$ and dimension $k$ is $k / n$. Two ternary linear codes are said to be equivalent if one can be obtained from the other by permutation of coordinate places and negation of some coordinate entries. A linear code is said to be isodual iff it is equivalent to its dual. Recall that a cyclic code of length $n$ over $\mathbb{F}_{q}$ can be regarded as an ideal in the principal ideal ring $\mathbb{F}_{q}[x] /\left(x^{n}-1\right)$. If $g(x)$ denote the generator polynomial of a cyclic code $C$, then the generator of the dual code, denoted by $h(x)$ is, up to sign, the reciprocal of its complement

$$
h(x)=\frac{x^{n}-1}{g(x)},
$$

where the reciprocal polynomial $f^{*}(x)$ of a polynomial $f(x)$, of degree $n$ over $\mathbb{F}_{3}$, is defined by

$$
f^{*}(x)=x^{n} f\left(\frac{1}{x}\right) .
$$

The elements of a code $C$ are called codewords, and the weight $w t(x)$ of a codeword $x$ is the number of positions where $x$ is nonzero. The Hamming distance $d(x, y)$ between two codewords $x$ and $y$ is $d(x, y)=w t(x-y)$. The minimum distance of a code $C$ is: 


$$
d(C)=\min \{d(x, y) / x, y \in C, x \neq y\} .
$$

If $C$ is linear then $d(C)$ equals the minimum weight of a nonzero codeword. The three parameters of a $q$-ary code are denoted by $[n, k, d]_{q}$ and are length, dimension, minimum distance. The so-called fundamental problem of coding theory is

- Find $d_{q}(n, k)$, the largest value of $d$ for which a code of parameters $[n, k, d]_{q}$ exists.

A code that attains this value is called an optimal code.

With the notation of the introduction we have, by definition,

$$
d_{C}(n) \leq d_{3}(n, n / 2)
$$

and

$$
d_{I}(n) \leq d_{F}(n) \leq d_{3}(n, n / 2) .
$$

\section{Isodual cyclic codes}

\subsection{Constructions}

We give seven constructions of isodual ternary cyclic codes and illustrate them by examples in the next section. We suppose that $n=2 m$ with $m$ odd and not a multiple of 3 . In that case the factorization

$$
x^{m}-1=(x-1) u(x) v(x)
$$

yields, by changing $x$ into $-x$ the factorization

$$
x^{m}+1=(x+1) u(-x) v(-x) .
$$

We choose

$$
g(x)=(x-1) u(x) v(-x) .
$$

We consider the following seven cases

(1) $u^{*}(x)=u(x), v^{*}(x)=v(x)$

(2) $u^{*}(x)=\epsilon v(x), v^{*}(x)=\eta u(x)$

(3) $u^{*}(x)=-v^{*}(x)$

(4) $u^{*}(x)=u(x), v^{*}(x)=v(-x)^{*}$

(5) $u^{*}(x)=u(-x)^{*}, v^{*}(x)=v(x)$

(6) $u^{*}(x)=u(x), v^{*}(x)=\eta v(-x)$

(7) $u^{*}(x)=\epsilon u(-x), v^{*}(x)=v(x)$

with $\epsilon, \eta= \pm 1$. 
Proposition 1 Keep the above notation In the seven cases above the cyclic code of generator $g(x)$ is isodual.

Proof In each case we compute the generator of the dual code. First

$$
\left(x^{n}-1\right) / g(x)=(x+1) u(-x) v(x) .
$$

Taking reciprocals of both sides, we obtain in the first five cases $\pm g(-x)$, and in the last two cases $[-g(-x)]^{*}$. The result follows.

\subsection{Table of values of $d_{I}(n)$ and $d_{C}(n)$}

In this table we note the different values of $d_{I}(n)$ and $d_{C}(n)$ respectively for isodual and cyclic codes of parameters $\left[n, \frac{n}{2}\right]_{3}$ according to the length of the code.

\begin{tabular}{lllllllllll}
\hline$n$ & 26 & 34 & 38 & 46 & 50 & 58 & 62 & 68 & 70 & 74 \\
$d_{I}(n)$ & 6 & 4 & 4 & 9 & 4 & 4 & 4 & & 14 & 14 \\
$d_{C}(n)$ & 8 & 4 & 4 & 13 & 4 & 4 & 4 & 8 & 14 & 14 \\
\hline
\end{tabular}

Remark For $n=34,38,50,58,62,70,74$ the minimum distance of the best isodual code of parameters $\left[n, \frac{n}{2}\right]_{3}$ is the same as that of the best cyclic code of rate one-half.

\section{Optimal cyclic codes of rate $1 / 2$ over $\mathbb{F}_{3}$}

4.1 Cyclic codes of parameters $[26,13]_{3}$

We begin our study of the minimum distance of ternary cyclic codes of parameters $\left[n, \frac{n}{2}\right], n$ even, and not a multiple of 3 . For linear codes the upper and lower bounds from [3] on $d_{3}(n, n / 2)$ coincide for $2 \leq n \leq 24$. For $n \geq 26$ (see [3]) the upper bounds are not always met. The table of bounds on $d_{3}(n, n / 2)$ for $26 \leq n \leq 74$ is given below.

\begin{tabular}{lllllllllll}
\hline $\mathrm{n}$ & $\mathbf{2 6}$ & 28 & 32 & $\mathbf{3 4}$ & $\mathbf{3 8}$ & 40 & 44 & $\mathbf{4 6}$ & $\mathbf{5 0}$ \\
$\mathrm{d}_{3}$ & $\mathbf{8 - 9}$ & $9-10$ & $10-11$ & $\mathbf{1 1 - 1 2}$ & $\mathbf{1 1 - 1 3}$ & $12-14$ & $13-15$ & $\mathbf{1 4 - 1 5}$ & $\mathbf{1 4 - 1 7}$ \\
\hline $\mathrm{n}$ & 52 & 56 & $\mathbf{5 8}$ & $\mathbf{6 2}$ & 64 & $\mathbf{6 8}$ & $\mathbf{7 0}$ & $\mathbf{7 4}$ \\
\hline $\mathrm{n}$ & 52 & & $16-18$ & $\mathbf{1 7 - 1 9}$ & $\mathbf{1 7 - 2 0}$ & $18-21$ & $\mathbf{1 6 - 2 2}$ & $\mathbf{1 7 - 2 3}$ & $\mathbf{1 8 - 2 4}$ \\
\hline $\mathrm{d}_{3}$ & $15-18$ & $16-2$ &
\end{tabular}

The algorithm of Chen as described in [6] allows us to derive the minimum distances of all ternary cyclic codes of length 26 and dimension 13 . The following decomposition into irreducible factors

$$
\begin{aligned}
x^{26}-1= & (1+x)(2+x)\left(1+2 x+x^{3}\right)\left(2+2 x+x^{3}\right)\left(2+x^{2}+x^{3}\right)\left(2+x+x^{2}+x^{3}\right) \\
& \left(1+2 x+x^{2}+x^{3}\right)\left(1+2 x^{2}+x^{3}\right)\left(1+x+2 x^{2}+x^{3}\right)\left(2+2 x+2 x^{2}+x^{3}\right)
\end{aligned}
$$


Table 1 Length 26

\begin{tabular}{|c|c|c|c|c|}
\hline $\mathrm{g}(\mathrm{x})$ & codeword(a) & {$\left[\begin{array}{l}u^{*}(x)= \\
v^{*}(x)=\end{array}\right.$} & {$\left[\frac{x^{26}-1}{g(x)}\right]^{*}=$} & wt(a) \\
\hline 10000000000001 & 10000000000001000000000000 & \multirow{6}{*}{$\begin{array}{l}{\left[\begin{array}{l}u^{*}(x)=v(x) \\
v^{*}(x)=u(x)\end{array}\right.} \\
{\left[\begin{array}{l}u^{*}(x)=v(x) \\
v^{*}(x)=u(x)\end{array}\right.} \\
{\left[\begin{array}{l}u^{*}(x)=u(x) \\
v^{*}(x)=-v(-x)\end{array}\right.}\end{array}$} & $g(-x)$ & 2 \\
\hline 22121212121211 & 21000000000002100000000000 & & $-g(-x)$ & 4 \\
\hline 11220102101001 & 10100000100001010000010000 & & \multirow[t]{4}{*}[-g(-x)]{$^{*}$} & 6 \\
\hline 20120100020121 & 12001010000002100202000000 & & & 8 \\
\hline 12112100012111 & 21000100000000200000000211 & & & 7 \\
\hline 22000102100211 & 22200000000102202020000000 & & & 8 \\
\hline 10020222110211 & 12010000000002102000000000 & \multirow{7}{*}{ 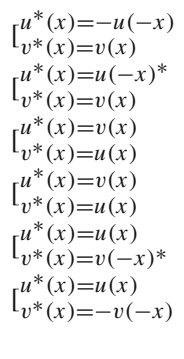 } & {$[-g(-x)]^{*}$} & 6 \\
\hline 12011200010121 & 10100000100001010000010000 & & $g(-x)$ & 6 \\
\hline 20000000000001 & 10000000000002000000000000 & & $-g(-x)$ & 2 \\
\hline 12222222222221 & 11000000000002200000000000 & & $g(-x)$ & 4 \\
\hline 10111211001201 & 22000000100001100000020000 & & $g(-x)$ & 6 \\
\hline 21120102202001 & 10100000100002020000020000 & & {$[-g(-x)]^{*}$} & 6 \\
\hline 12121021020221 & 10200001000002000012000200 & & & 7 \\
\hline
\end{tabular}

over $\mathbb{F}_{3}$ comprizes 8 polynomials of degree 3 and two linear polynomials. Thus there are $\left(\begin{array}{l}8 \\ 4\end{array}\right)\left(\begin{array}{l}2 \\ 1\end{array}\right)=140$ possible generators polynomials of degree $3 \times 4+1=13$. Some of these polynomials along with a minimum weight codeword $a$ and its weight are recorded in Table 1.

Remark There are 54 codes with the optimal minimum distance 8 among the 140 cyclic codes of parameters $[26,13]_{3}$.

We summarize our first experimental result by

Proposition 2 We have $d_{C}(26)=8$. Note that $d_{3}(26,13) \in\{8,9\}$, by $[3]$.

4.2 Cyclic codes of parameters $\left[n, \frac{\mathbf{n}}{\mathbf{2}}\right]_{3}$ where $n=34,38,50,58,62$

- In the cases $n=34,38,58,62$, for the all cyclic codes of parameters $\left[n, \frac{n}{2}\right]_{3}$, we have

$$
\begin{aligned}
x^{n}-1= & (1+x)\left(1+x+x^{2}+\cdots+x^{\frac{n}{2}-2}+x^{\frac{n}{2}-1}\right) \\
& (2+x)\left(1+2 x+x^{2}+\cdots+2 x^{\frac{n}{2}-2}+x^{\frac{n}{2}-1}\right) .
\end{aligned}
$$

Thus there are four choices for the generator polynomial of each code. 
For the cyclic codes of parameters $[50,25]_{3}$, we have 8 possible choices for the generator polynomial $g(x)$ of degree 25 .

$$
\begin{aligned}
x^{50}-1= & (1+x)(2+x)\left(1+x+x^{2}+x^{3}+x^{4}\right)\left(1+2 x+x^{2}+2 x^{3}+x^{4}\right) \\
& \left(1+x^{5}+x^{10}+x^{15}+x^{20}\right)\left(1+2 x^{5}+x^{10}+2 x^{15}+x^{20}\right) .
\end{aligned}
$$

But in all cases, the maximal minimum distance $d_{C}$ of such codes is equal to 4 , and we have always:

$$
\left[\begin{array}{c}
u^{*}(x)=u(x) \\
v^{*}(x)=v(x)
\end{array} \text { with }\left[\frac{x^{n}-1}{g(x)}\right]^{*}= \pm g(-x)\right.
$$

Proposition 3 For $n=34,38,50,58,62$ the cyclic codes of parameters $[n, n / 2]_{3}$ are isodual.

\subsection{Cyclic codes of parameters $[46,23]_{3}$}

For cyclic codes of parameters [46, 23], the factorization of $x^{46}-1$ yields $12=2 \times\left(\begin{array}{l}4 \\ 2\end{array}\right)$ possible generator polynomials of degree $23=1+2 \times 11$.

$$
\begin{aligned}
x^{46}-1= & (1+x)(2+x)\left(1+2 x+x^{2}+x^{3}+2 x^{4}+x^{6}+x^{8}+x^{11}\right) \\
& \left(2+2 x+2 x^{2}+x^{3}+x^{4}+2 x^{6}+2 x^{8}+x^{11}\right) \\
& \left(2+x^{3}+x^{5}+2 x^{7}+2 x^{8}+x^{9}+x^{10}+x^{11}\right) \\
& \left(1+x^{3}+x^{5}+2 x^{7}+x^{8}+x^{9}+2 x^{10}+x^{11}\right) .
\end{aligned}
$$

The analogue of Table 1 is Table 2 below.

For the isodual cyclic codes $[46,23]_{3}$ we have:

$$
\left[\begin{array}{l}
u^{*}=\epsilon v \\
v^{*}=\eta u
\end{array} ; \epsilon, \quad \eta= \pm 1\right.
$$

or

$$
u^{*}(x)=-v^{*}(x)
$$

with

$$
\left[\frac{x^{46}-1}{g(x)}\right]^{*}= \pm g(-x) \text {. }
$$

The optimum distance of such a cyclic codes is

Proposition 4 We have $d_{C}(46)=13$. Note that $d_{3}(46,23) \in\{14,15\}$. 


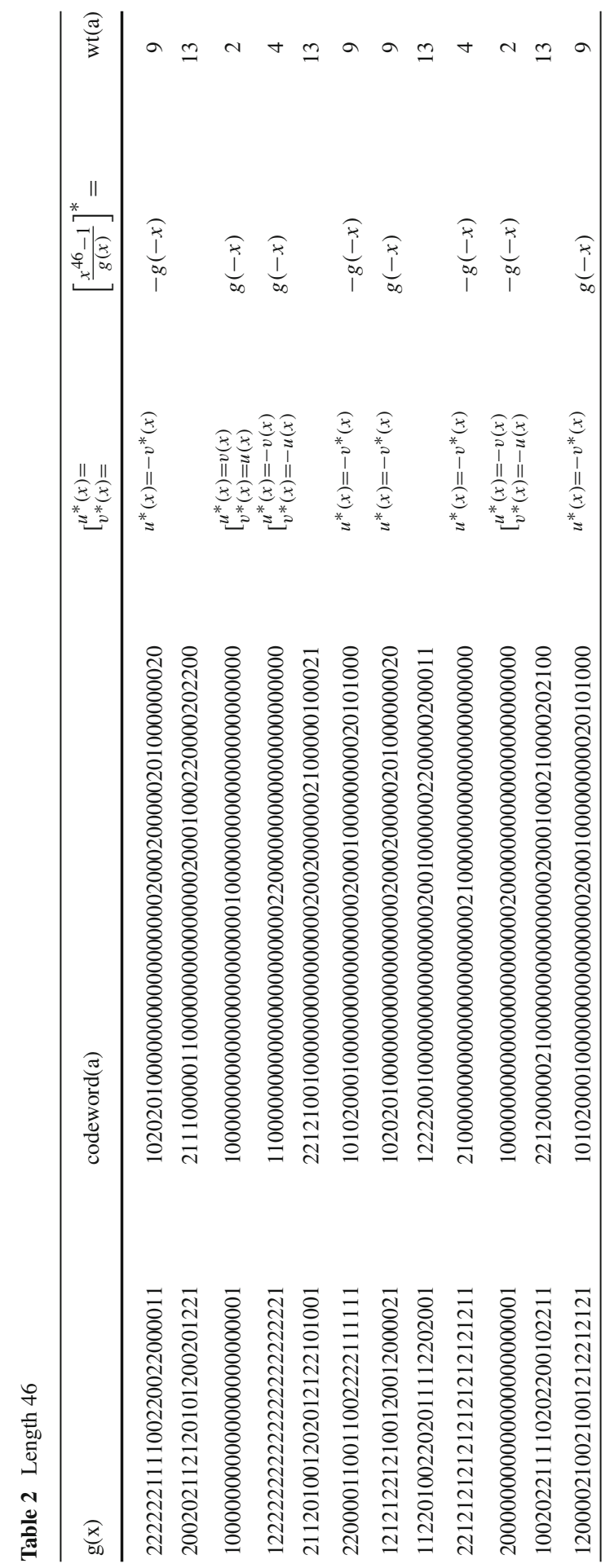


Table 3 Length 68

\begin{tabular}{|c|c|c|c|}
\hline $\mathrm{g}(\mathrm{x})$ & {$\left[\begin{array}{l}u^{*}(x)= \\
v^{*}(x)=\end{array}\right.$} & {$\left[\frac{x^{68}-1}{g(x)}\right]^{*}=$} & wt(a) \\
\hline 20101221001001021002021120020020121 & $220 \ldots 0110 \ldots 0220 \ldots 0110 \ldots 0$ & & 8 \\
\hline 20201020102010201020102010201020101 & $2010 \ldots .00 \ldots 02010 \ldots .00 \ldots . .0$ & & 4 \\
\hline 22201002001122020022010020011220201 & $210 \ldots 0210 \ldots 0210 \ldots 0210 \ldots 0$ & & 8 \\
\hline 21201001001221010021020020021120201 & $220 \ldots 0110 \ldots 0220 \ldots 0110 \ldots 0$ & & 8 \\
\hline 20000000000000000000000000000000001 & 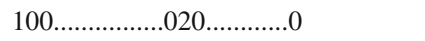 & & 2 \\
\hline 20101122001002011001011220010020111 & $210 \ldots 0210 \ldots 0210 \ldots 0210 \ldots 0$ & & 8 \\
\hline 10102121002012120021201012202101021 & $20 \ldots . .010 \ldots .020 \ldots .010 \ldots \ldots 0$ & & 4 \\
\hline 11020220211010222001111020022220101 & $10 \ldots .010 \ldots . .010 \ldots \ldots 010 \ldots . .0$ & & 4 \\
\hline 12010120221010212002121020012120101 & $20 \ldots \ldots \ldots 010 \ldots 020 \ldots 010 \ldots 0$ & & 4 \\
\hline 10202020202020202020202020202020201 & $1010 \ldots \ldots \ldots 02020 \ldots \ldots \ldots \ldots \ldots$ & & 4 \\
\hline 10102222002011110022201011202202011 & $10 \ldots .010 \ldots 010 \ldots \ldots .010 \ldots .0$ & & 4 \\
\hline 10000000000000000000000000000000001 & 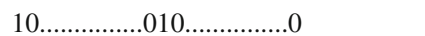 & & 2 \\
\hline
\end{tabular}

\subsection{Cyclic codes of parameters $[68,34]_{3}$}

Likewise the factorization of $x^{68}-1$ yields 12 possibilities for the generator polynomial of the code.

$$
\begin{aligned}
x^{68}-1= & (1+x)(2+x)\left(1+x^{2}\right)\left(1+2 x+2 x^{4}+2 x^{5}+2 x^{6}+2 x^{10}\right. \\
& \left.+x^{11}+2 x^{12}+x^{15}+x^{16}\right)\left(1+x+x^{2}+x^{3}+x^{4}+x^{5}+x^{6}\right. \\
& \left.+x^{7}+x^{8}+x^{9}+x^{10}+x^{11}+x^{12}+x^{13}+x^{14}+x^{15}+x^{16}\right) \\
& \left(1+x+2 x^{4}+x^{5}+2 x^{6}+2 x^{10}+2 x^{11}+2 x^{12}+2 x^{15}+x^{16}\right) \\
& \left(1+2 x+x^{2}+2 x^{3}+x^{4}+2 x^{5}+x^{6}+2 x^{7}+x^{8}+2 x^{9}+x^{10}\right. \\
& \left.+2 x^{11}+x^{12}+2 x^{13}+x^{14}+2 x^{15}+x^{16}\right) .
\end{aligned}
$$

The analogue of Table 1 is Table 3 .

If we take all $g(x)$ that divide $x^{68}-1$, as indicated in Table 4, we have always:

$$
\left[\frac{x^{68}-1}{g(x)}\right]^{*} \neq \pm g(-x)
$$

and

$$
\left[\frac{x^{68}-1}{g(x)}\right]^{*} \neq[-g(-x)]^{*} .
$$

This shows that the isodual cyclic codes of parameters $[68,34]_{3}$ are not isodual by the constructions of the preceding section.

Proposition 5 We have $d_{C}(68)=8$. Note that $d_{3}(68,34) \geq 16$. 


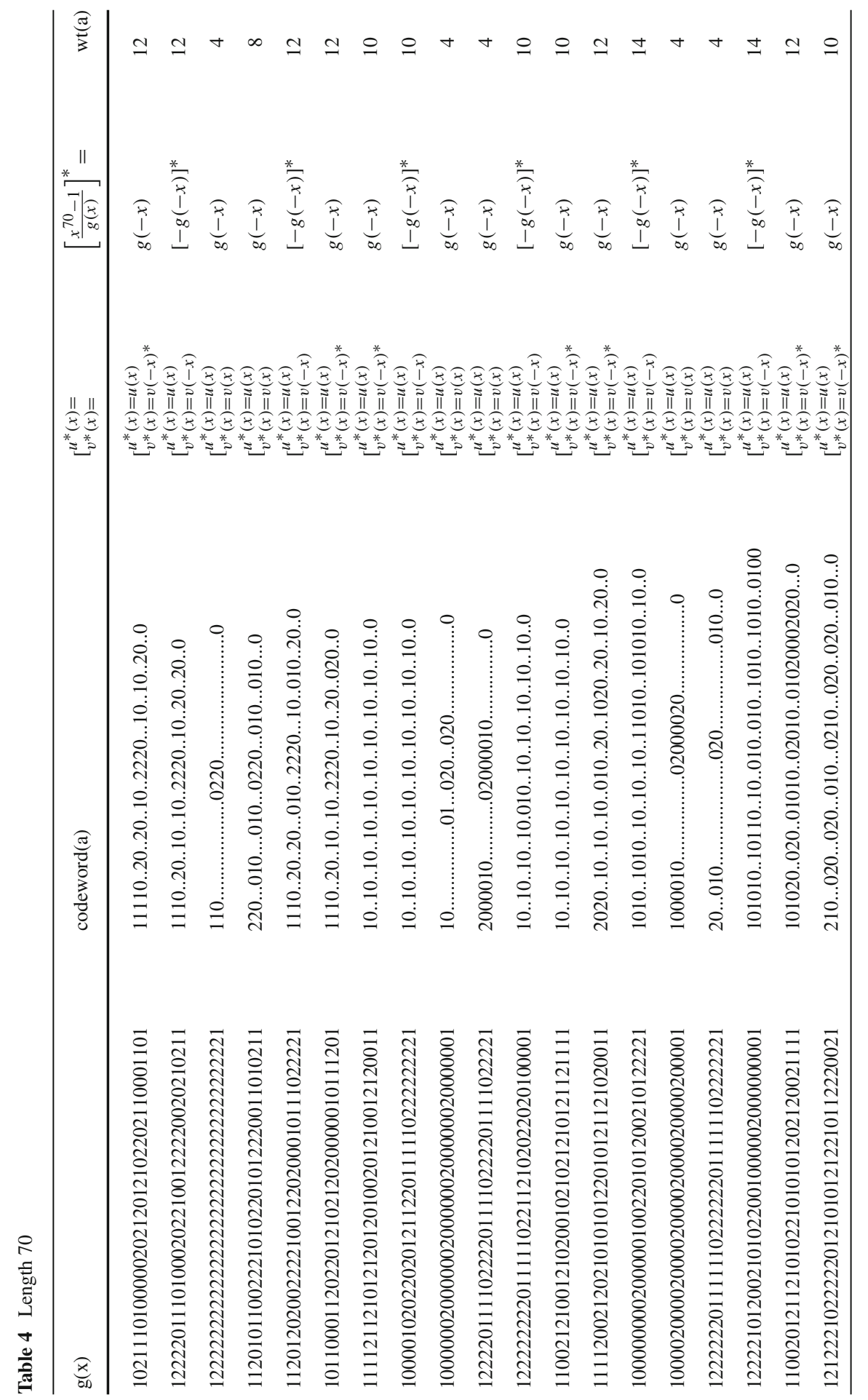




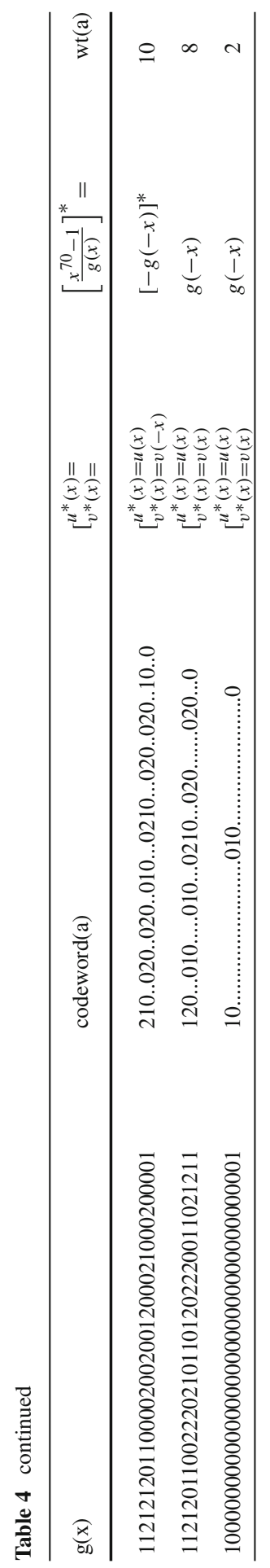


4.5 Cyclic codes of parameters $[70,35]_{3}$

The factorization of $x^{70}-1$ yields 48 possibilities for the generator polynomial $g(x)$ of degree 35 of the code.

$$
\begin{aligned}
x^{70}-1= & (1+x)(2+x)\left(1+x+x^{2}+x^{3}+x^{4}\right)\left(1+2 x+x^{2}+2 x^{3}+x^{4}\right) \\
& \left(1+x+x^{2}+x^{3}+x^{4}+x^{5}+x^{6}\right)\left(1+2 x+x^{2}+2 x^{3}+x^{4}+2 x^{5}+x^{6}\right) \\
& \left(1+2 x+2 x^{2}+x^{3}+2 x^{4}+x^{5}+x^{7}+2 x^{8}+x^{10}+x^{12}\right) \\
& \left(1+x+2 x^{2}+2 x^{3}+2 x^{4}+2 x^{5}+2 x^{7}+2 x^{8}+x^{10}+x^{12}\right) \\
& \left(1+x^{2}+2 x^{4}+2 x^{5}+2 x^{7}+2 x^{8}+2 x^{9}+2 x^{10}+x^{11}+x^{12}\right) \\
& \left(1+x^{2}+2 x^{4}+x^{5}+x^{7}+2 x^{8}+x^{9}+2 x^{10}+2 x^{11}+x^{12}\right) .
\end{aligned}
$$

For this codes we have 3 cases:

$$
\left[\begin{array} { l } 
{ u ^ { * } ( x ) = u ( x ) } \\
{ v ^ { * } ( x ) = v ( x ) }
\end{array} \text { or } \left[\begin{array}{l}
u^{*}(x)=u(x) \\
v^{*}(x)=v(-x)^{*}
\end{array} \text { with }\left[\frac{x^{70}-1}{g(x)}\right]^{*}= \pm g(-x)\right.\right.
$$

or

$$
\left[\begin{array}{l}
u^{*}(x)=u(x) \\
v^{*}(x)=v(-x)
\end{array} \text { with }\left[\frac{x^{70}-1}{g(x)}\right]^{*}=[-g(-x)]^{*} .\right.
$$

We summarize the parameters of the cyclic codes $[70,35]_{3}$ in Tables 4 and 5 below.

Remark The cyclic codes of parameters $[70,35]_{3}$ are all isodual.

Proposition 6 The optimal minimum distance of the cyclic codes of parameters $[70,35]_{3}$ is $d_{C}(70)=14$.

\subsection{Cyclic codes of parameters $[74,37]_{3}$}

The determination of the minimum distance for these codes required in some cases up to 12 hours of CPU time on a PC. The factorisation of $x^{74}-1$ yields 12 possible choices for a generator polynomial of degree 37.

$$
\begin{aligned}
x^{74}-1= & (1+x)(2+x)\left(1+2 x^{2}+2 x^{4}+x^{5}+2 x^{7}+2 x^{11}+x^{13}+2 x^{14}+2 x^{16}\right. \\
& \left.+x^{18}\right)\left(1+2 x^{2}+2 x^{4}+2 x^{5}+x^{7}+x^{11}+2 x^{13}+2 x^{14}+2 x^{16}+x^{18}\right) \\
& \left(1+x+2 x^{2}+2 x^{3}+x^{4}+2 x^{5}+2 x^{6}+2 x^{8}+2 x^{9}+2 x^{10}+2 x^{12}\right. \\
& \left.+2 x^{13}+x^{14}+2 x^{15}+2 x^{16}+x^{17}+x^{18}\right)\left(1+2 x+2 x^{2}+x^{3}+x^{4}\right. \\
& +x^{5}+2 x^{6}+2 x^{8}+x^{9}+2 x^{10}+2 x^{12}+x^{13}+x^{14}+x^{15}+2 x^{16} \\
& \left.+2 x^{17}+x^{18}\right) .
\end{aligned}
$$

The analogue of Table 1 is Table 6.

Proposition 7 We have $d_{C}(74)=14$. 


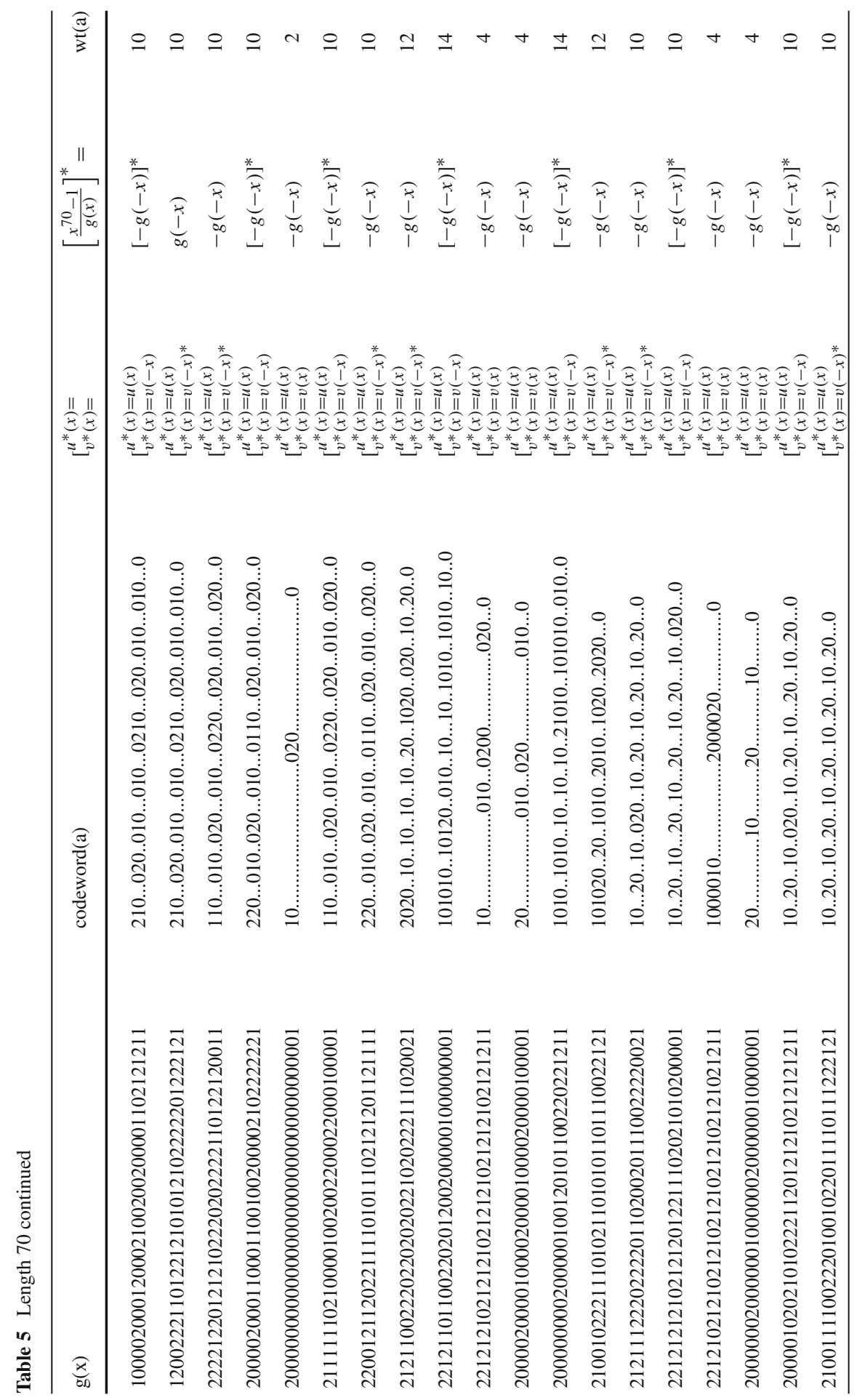




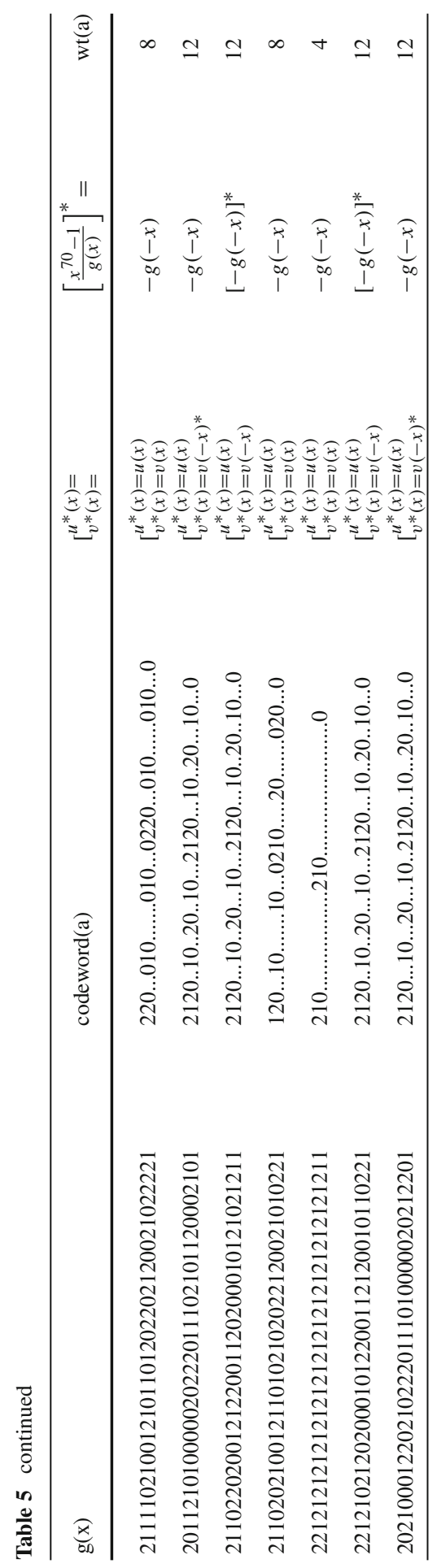




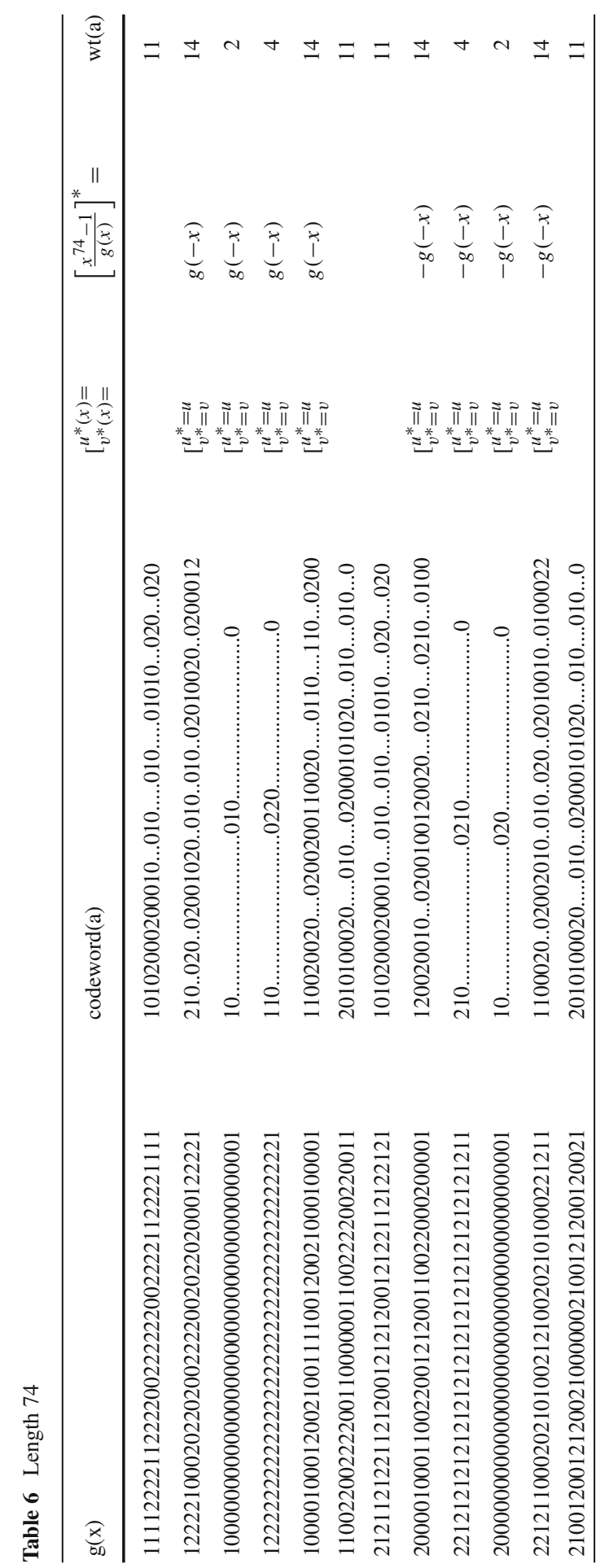


Acknowledgments The authors thanks the referees for helpful suggestions that greatly improved the presentation of the material. They are very grateful to $\mathrm{H}$. Aissaoui (Telecom ParisTech) for the optimization of the research algorithm of the minimum distance of a cyclic codes $C[26,13]_{3}$.

Open Access This article is distributed under the terms of the Creative Commons Attribution License which permits any use, distribution, and reproduction in any medium, provided the original author(s) and the source are credited.

\section{References}

1. Cary Huffman, W., Pless, V.: Fundamentals of Error Correcting Codes. Cambridge University Press, Cambridge (2003)

2. Dougherty, S.T., Gulliver, T.A., Harada, M.: Optimal ternary formally self-dual codes. Discrete Math. 196, 117-135 (1999)

3. Grassl, M.: Bounds on the minimum distance of linear codes (Electronic table; online). http://www. codetables.de.win/math/dw/voorlincood.html

4. MacWilliams, F.J., Sloane, N.J.A.: The Theory of Error-Correcting Codes. North-Holland, Amsterdam (1977)

5. Rains, E.M., Sloane, N.J.A.: Self-dual codes. In: Pless, V.S., Huffman, W.C. (eds.) Handbook of Coding Theory. Elsevier, Amsterdam (1998)

6. Voloch, J.F.: Computing the minimal distance of cyclic codes. Comp. Appl. Math. 24, 393-398 (2005) 